\title{
Root and root canal morphology of the permanent dentition in a Caucasian population: a cone-beam computed tomography study
}

\author{
J. N. R. Martins ${ }^{1,2}$ (D), D. Marques ${ }^{2,3,4}$, A. Mata ${ }^{4,5}$ \& J. Caramês $^{2,4,6}$ \\ ${ }^{1}$ Department of Endodontics, Faculdade de Medicina Dentária, Universidade de Lisboa, Lisboa; ${ }^{2}$ Implantology Institute, \\ Lisboa; ${ }^{3}$ Department of Anatomy and Physiology, Faculdade de Medicina Dentária, Universidade de Lisboa, Lisboa; \\ ${ }^{4}$ LIBPhys-FCT UID/FIS/04559/2013, Lisboa; ${ }^{5}$ Department of Oral Biology, Faculdade de Medicina Dentária, Universidade de \\ Lisboa, Lisboa; and ${ }^{6}$ Oral Surgery Department, Faculdade de Medicina Dentária, Universidade de Lisboa, Lisboa, Portugal
}

\begin{abstract}
Martins JNR, Marques D, Mata A, Caramês J. Root and root canal morphology of the permanent dentition in a Caucasian population: a cone-beam computed tomography study. International Endodontic Journal.
\end{abstract}

Aim To investigate in vivo the number of roots, the configuration of the root canal system and the root canal splitting and merging levels in the permanent dentition of a Caucasian population.

Methodology A total of 11892 teeth were inspected in cone-beam computed tomography examinations that were collected from a pre-existing database. The number of roots was identified, and canal configurations were classified according to Vertucci's classification and its supplemental configurations. In addition, the merging and separation positions along the length of the root canal were identified. The Z-test was used to analyse the differences between independent groups. A value of $P<0.05$ was considered significant.

Results The majority of tooth types had considerable variation in the number of roots and root canal configuration types. Radix entomolaris and paramolaris were rare occurrences. The mesiobuccal root of the maxillary first and second molars had two root canals in $71 \%$ and $44 \%$ of cases, respectively. Nearly $30 \%$ of the mandibular incisors had two root canals. Root canal merging and splitting appeared more often in mandibular teeth. Merging was more common in the middle third of the root canal in the maxillary teeth and in the middle and apical thirds in mandibular teeth. Root canal splitting did not exhibit a tendency, and their position along the length of the root canal varied from tooth to tooth.

Conclusion Clinicians must be aware that each tooth may display several types of root canal configuration. However, a greater variability in root canal configuration was found in maxillary second premolars and in the mesiobuccal roots of maxillary molars. Main root canal merging and splitting may also be expected to occur at any level of the root canal.

Keywords: anatomy, cone-beam computed tomography, morphology, root canal.

Received 12 April 2016; accepted 21 November 2016

\section{Introduction}

Knowledge about the average number of root canals in a specific root is as important as an understanding

Correspondence: Jorge N. R. Martins, Instituto de Implantologia of Lisbon, Av.Columbano Bordalo Pinheiro, $50-5^{\circ}$ e $6^{\circ}$, 1070-064 Lisboa, Portugal (Tel.: +351 9652640 63, e-mail: jnr_martins@yahoo.com.br). of the relationships between them. Clinically, it is important to know that a root has a single root canal at the pulp chamber floor that splits into multiple canals at a certain point of the root canal length; it is also important to determine the level of the root canal where the separation occurs. Root canal splitting in the middle and apical root canal sections may go unnoticed, is more difficult to manage and may stress endodontic instruments. Information on the position of merging and splitting of the main root canal is not 
fully available. Studies have reported the presence of transverse anastomosis/intercanal communications (Vertucci 1984, Çaliskan et al. 1995, Alavi et al. 2002, Gulabivala et al. 2002, Sert \& Bayirli 2004) and have analysed their prevalence according to root canal level. The awareness of these anastomoses and isthmi is important (Estrela et al. 2015b) but may be limited if a clinician initiates root canal treatment and needs to know the most common point in the root canal system at which a root canal is likely to split into multiple canals. Two studies that clearly describe the position of root canal merging and the distribution of their position are those from Zhang et al. (2014) (using microcomputed tomography [micro-CT]) and Martins et al. (2016) (using cone-beam computed tomography $[\mathrm{CBCT}]$ ); however, these studies only presented information for teeth with fused roots. No information is available for nonfused roots or for the main position where root canals split.

The objective of this investigation was to analyse in vivo, using cone-beam computed tomography the number of roots, the canal system configuration and the root canal fusion levels (splitting and merging) in the permanent human dentition.

\section{Materials and methods}

A total of 11892 teeth were included (Table 1). The sample was obtained from 646 patients (228 males and 418 females) with a mean age of 51 years. All teeth were included. Teeth with endodontic treatments, immature apices, root resorptions or teeth that could not be correctly evaluated due to image artefacts or incorrect techniques were excluded, as were third molars. The CBCT examinations were performed between May 2011 and January 2016 and were collected from the database in the Radiology Department of a health centre in Lisbon, Portugal. The data were analysed retrospectively by a single observer from January 2015 to January 2016 after approval of the study by the ethics commission of the Instituto de Implantologia. All of the examinations were performed for diagnostic purposes prior to complex oral surgery procedures and were obtained using a large field of view (FOV) at a $0.20 \mathrm{~mm}$ voxel size, $80 \mathrm{kV}$ and $15 \mathrm{~mA}$, within an exposure time of 12 seconds using a Planmeca scanner (Planmeca Promax, Planmeca, Finland), according to the manufacturer's instructions. The images were analysed using computer visualization software (Planmeca Romexis, Planmeca). Cross-sectional images were reconstructed as 0.6-mm-thick slices, and a noise filter and artefact reduction were applied. All of the teeth under evaluation were analysed in three planes (coronal, sagittal and axial). The evaluation was performed scrolling through the CBCT volume until an understanding of the anatomy was achieved.

Teeth were classified according to the following parameters:

\section{Number of roots}

2. Root canal system configuration (Vertucci's classification and supplemental configurations). In the maxillary and mandibular molars, the usual three and two roots, respectively, were classified separately due to the impracticability of classifying them together. However, if there was any type of root fusion, these teeth were classified as 'fused roots', and no further analysis was performed. For all of the other groups of teeth, classification was performed for the tooth as a whole, independent of the number of roots, following the methodology of Vertucci (1984).

3. Merging and separation level of the root canals if a root canal fusion was present. Using the software ruler, each root was divided into three-thirds: a coronal section (from the cementoenamel junction to $1 / 3$ of the root length), a middle section (from $1 / 3$ to $2 / 3$ of the root length) and an apical section (from $2 / 3$ of the root length to the radiographic apex).

\section{Statistical analysis}

All of the teeth were classified according to their anatomy, and the data were analysed using SPSS software (IBM SPSS Statistics, Version 22, Chicago, IL, USA) from which absolute counts and proportions for the analysed groups were extracted. The primary outcomes were the root canal configuration and the number of roots. The percentage of each group was calculated, as was the range for the true population proportion, to a confidence level of 95\%. The Z-test for proportions was used to analyse the differences in independent subgroups. For all of the compared groups, a $P$ value of $<0.05$ was considered significant.

The Cohen's kappa test was used to determine the intrarater reliability, a single observer performed the evaluation of 527 teeth twice (which represents $4.34 \%$ of the total sample), with 1 month between 
observations. The kappa coefficient of agreement between both evaluations was $89.2 \%$ with an asymptotic standard error of $\pm 1.9 \%$.

\section{Results}

\section{Number of roots}

Tables 1 and 2 summarize the number of roots in each group of teeth. The maxillary first and second molars had three roots in 91.1\% [88.7-93.5\% CI 95\%] and 72.9\% [69.78-76.02\% CI 95\%] of cases, respectively. The difference in the number of roots between the maxillary molars was significant $(P<0.05)$. The mandibular first and second molars had two roots in 97.1\% [95.55-98.65\% CI 95\%] and $83.1 \%$ [80.26-85.94\% CI 95\%] of cases, respectively. The difference in the number of roots between the mandibular molars was significant $(P<0.05$; Table 1).

In the mandibular first and second molars, the prevalence of a third root was $2.2 \%$ [0.84-3.56\% CI 95\%] and 2.7\% [1.47-3.93\% CI 95\%], respectively. The difference in the number of third roots between mandibular molars was nonsignificant $(P>0.05)$. A radix entomolaris was present in 10 cases in the first mandibular molars $(2.2 \%$ [0.84-3.56\% CI 95\%]) and in four cases in the mandibular second molar $(0.6 \%$ [0.01-1.19\% CI 95\%]; Fig. 1). The difference in the presence of radix entomolaris between the mandibular molars was significant $(P<0.05$; Table 2$)$.

\section{Root canal system classification}

Table 3 summarizes the root canal system configuration for maxillary teeth. The tooth with the most unpredictable anatomy was the maxillary second premolar. The most common root canal system configuration was Type I (1-1), but it was only present in $39.4 \%$ [35.46-43.34\% CI 95\%] of cases. The most common configuration for the maxillary first premolar was Type IV (2-2), with a prevalence of $68.0 \%$ [64.52-71.48\% CI 95\%], but eleven other types of configurations were found for this tooth (Fig. 2). The prevalence of two root canal configurations (all of the types combined) in the mesiobuccal root of the maxillary first and second molars with three independent roots was $71.05 \% \quad[67.05-75.05 \%$ CI 95\%] and $43.56 \%$ [39.48-47.64\% CI 95\%], respectively. The occurrence of two root canals systems in the mesiobuccal root of the maxillary first molar was significantly higher when compared to the second molar $(P<0.05)$.

Table 4 summarizes the root canal morphology for mandibular teeth. Two root canals in both the mandibular central and lateral incisors were present in approximately $30 \%$ of cases (Fig. 3). The mesial roots of double-rooted mandibular first molars had two root canals in 93.4\% [91.07-95.73\% CI 95\%] of cases, and three root canals were present in $5.5 \%$ of cases [3.36-7.64\% CI 95\%] (Fig. 4). The mandibular second molar had a Type II (2-1) configuration in the mesial root in $63.9 \%$ [59.9-67.9\% CI

Table 1 Number of teeth in each group and the respective distribution of the number of roots

\begin{tabular}{|c|c|c|c|c|c|}
\hline & \multirow{2}{*}{$\begin{array}{c}\text { Total } \\
\text { Sample }(n)\end{array}$} & \multicolumn{4}{|c|}{ Number of roots } \\
\hline & & 1 & 2 & 3 & 4 \\
\hline Maxillary central incisor & $872(100 \%)$ & $872(100 \%)$ & - & - & - \\
\hline Maxillary lateral incisor & $902(100 \%)$ & $902(100 \%)$ & - & - & - \\
\hline Maxillary canine & $962(100 \%)$ & $962(100 \%)$ & - & - & - \\
\hline Maxillary 1st premolar & $690(100 \%)$ & $336(48.7 \%)$ & $339(49.1 \%)$ & $15(2.2 \%)$ & - \\
\hline Maxillary 2nd premolar & $591(100 \%)$ & $558(94.4 \%)$ & $33(5.6 \%)$ & - & - \\
\hline Maxillary 1st molar & $542(100 \%)$ & $3(0.6 \%)$ & $45(8.3 \%)$ & $494(91.1 \%)$ & - \\
\hline Maxillary 2nd molar & $778(100 \%)$ & $104(13.4 \%)$ & $103(13.2 \%)$ & $567(72.9 \%)$ & $4(0.5 \%)$ \\
\hline Mandibular central incisor & $1160(100 \%)$ & $1160(100 \%)$ & - & - & - \\
\hline Mandibular lateral incisor & $1191(100 \%)$ & $1191(100 \%)$ & - & - & - \\
\hline Mandibular canine & $1200(100 \%)$ & $1166(97.2 \%)$ & $34(2.8 \%)$ & - & - \\
\hline Mandibular 1st premolar & $1054(100 \%)$ & $1052(99.8 \%)$ & $2(0.2 \%)$ & - & - \\
\hline Mandibular 2nd premolar & $833(100 \%)$ & 832 (99.9\%) & $1(0.1 \%)$ & - & - \\
\hline Mandibular 1st molar & $450(100 \%)$ & $3(0.7 \%)$ & $437(97.1 \%)$ & $10(2.2 \%)$ & - \\
\hline Mandibular 2nd molar & $667(100 \%)$ & $95(14.2 \%)$ & $554(83.1 \%)$ & $18(2.7 \%)$ & - \\
\hline Total & $11892(100 \%)$ & & & & \\
\hline
\end{tabular}


Table 2 Characteristics of the maxillary and mandibular molars with extra roots

\begin{tabular}{|c|c|c|c|}
\hline Teeth $(n=32)$ & Gender & Number of roots & Configuration of the extra root \\
\hline $\begin{array}{l}\text { Maxillary 2nd } \\
\text { molar }(n=4)\end{array}$ & Male (4), Female (-) & 4 & $\begin{array}{l}2 \times \text { One MB root splitting in two at the middle portion } \\
\text { of the root (Type } 1-2 \text { ) } \\
2 \times \text { Two palatal roots (radix mesiolingualis) }\end{array}$ \\
\hline $\begin{array}{l}\text { Mandibular 1st } \\
\text { molar }(n=10)\end{array}$ & Male (5), Female (5) & 3 & $10 \times$ Extra distolingual root (radix entomolaris) \\
\hline $\begin{array}{l}\text { Mandibular } 2 \text { nd } \\
\text { molar }(n=18)\end{array}$ & Male (7), Female (11) & 3 & $\begin{array}{l}7 \times \text { Mesial root is divided into two similar mesial roots, } \\
\text { both with Type } 1-1 \\
4 \times \text { Normal distal root with an extra distolingual root } \\
\text { (radix entomolaris) } \\
4 \times \text { Normal mesial root with an extra mesiolingual root } \\
3 \times \text { Normal mesial root with an extra mesiobuccal root } \\
\text { (radix paramolaris) }\end{array}$ \\
\hline
\end{tabular}
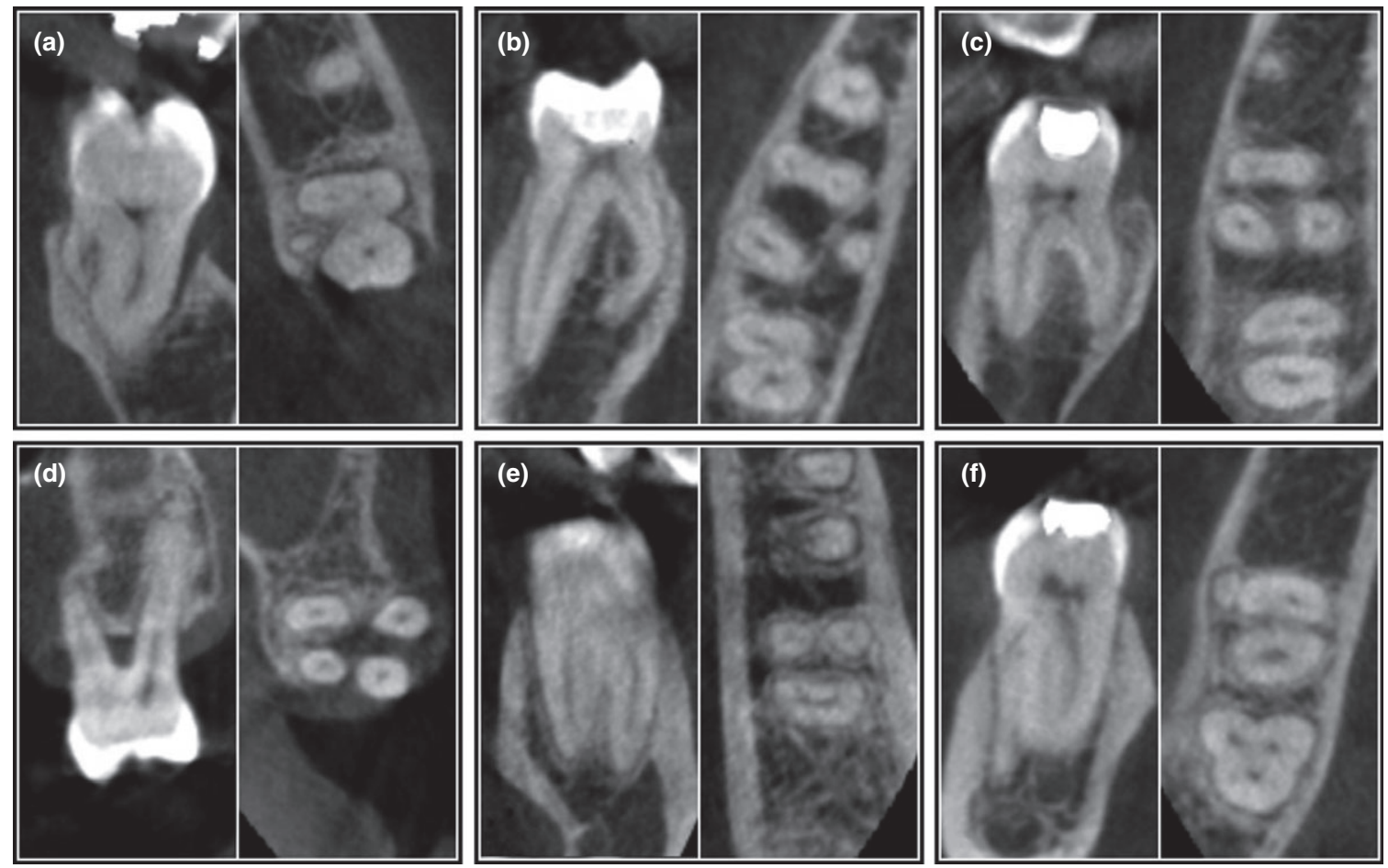

Figure 1 Examples of extra root morphologies. (a-c) Radix entomolaris configuration in the mandibular molars, (d) radix mesiolingualis in a maxillary second molar, (e) mesial root divided into two similar mesial roots in a mandibular second molar, (f) radix paramolaris in a mandibular second molar.

95\%] of the samples. The distal root of the mandibular first and second molars had a single root canal in $70.9 \% \quad[66.64-75.16 \%$ CI $95 \%]$ and $93.5 \%$ [91.45-95.55\% CI 95\%] of cases, respectively. This difference in the distal root was significant $(P<0.05)$.

\section{Splitting and merging levels}

Table 5 summarizes the main root canal splitting and merging levels of maxillary teeth. The maxillary tooth with the highest prevalence of root canal splitting was the second premolar (14.2\% [11.39-17.01\% CI 


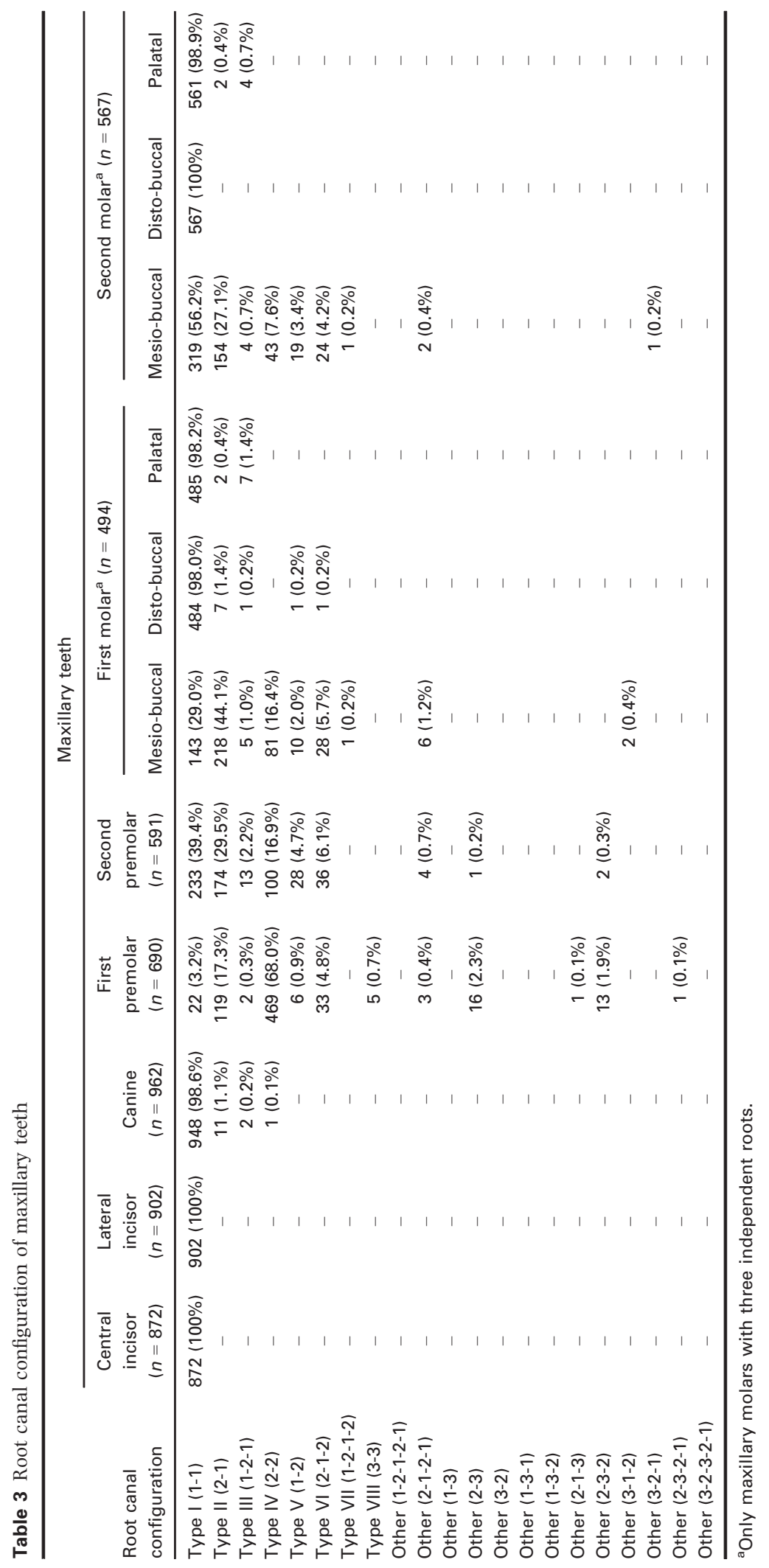



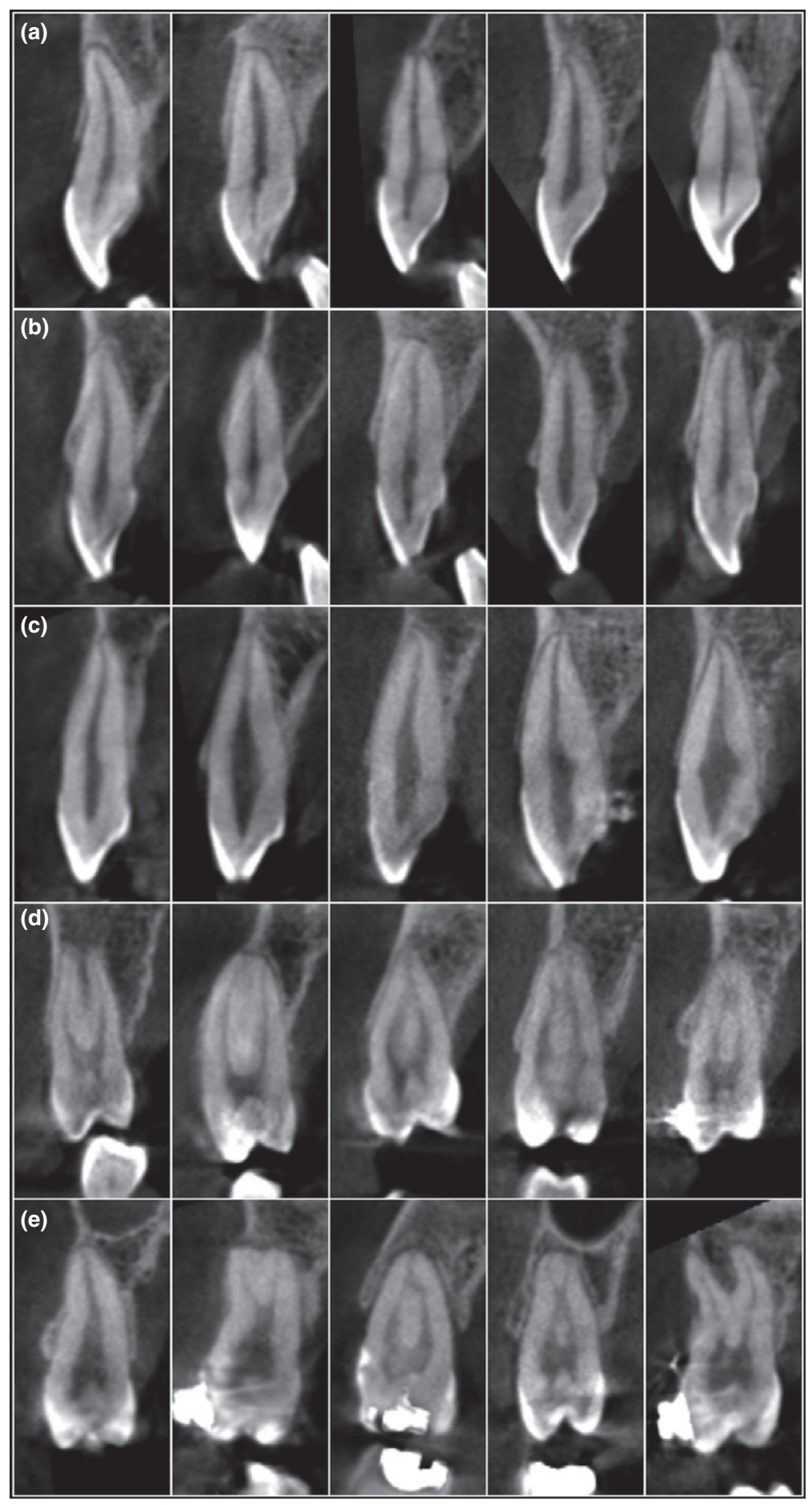

Figure 2 Examples of several root canal system configurations on maxillary teeth. (a) Central incisors, (b) lateral incisors, (c) canine, (d) first premolar, (e) second premolar. 


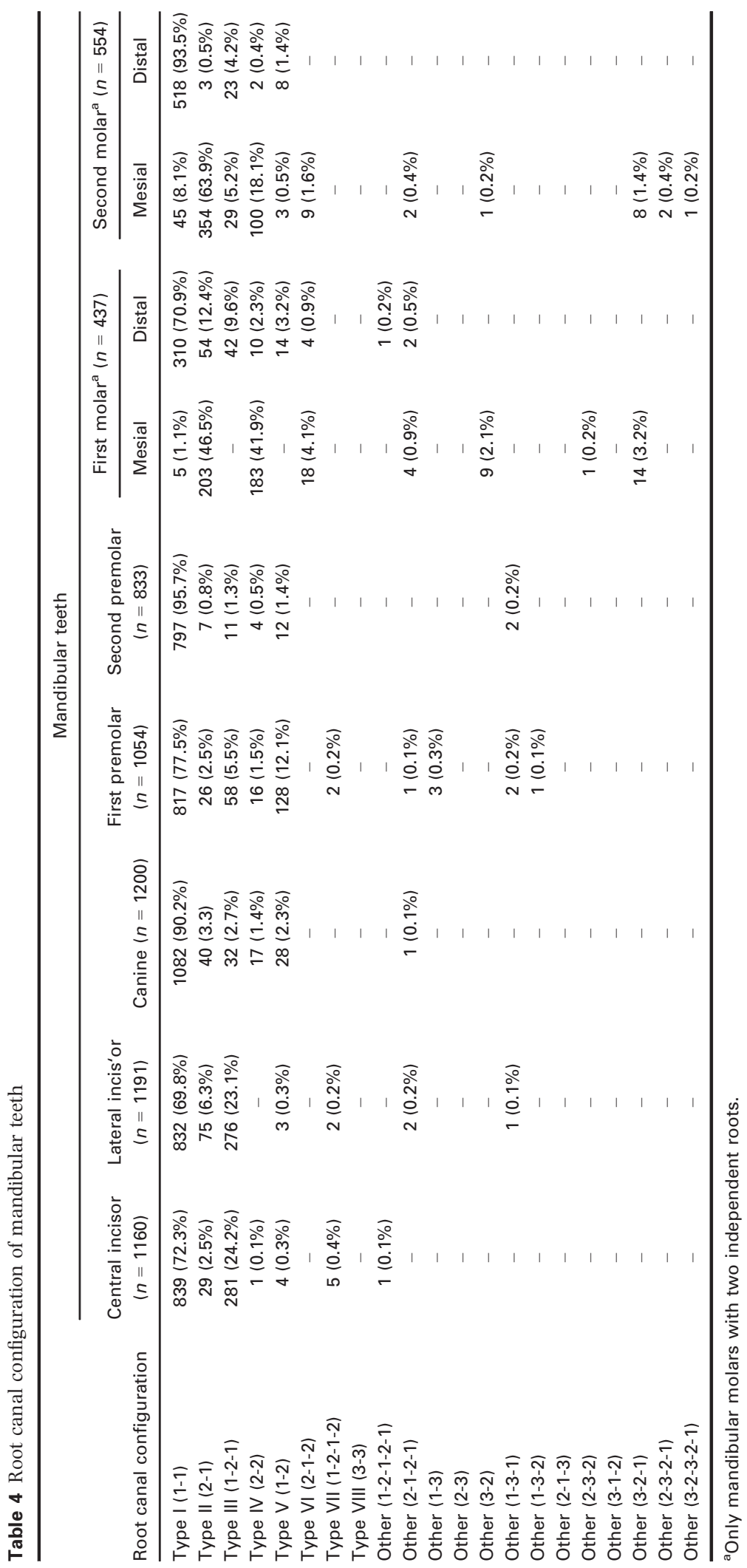



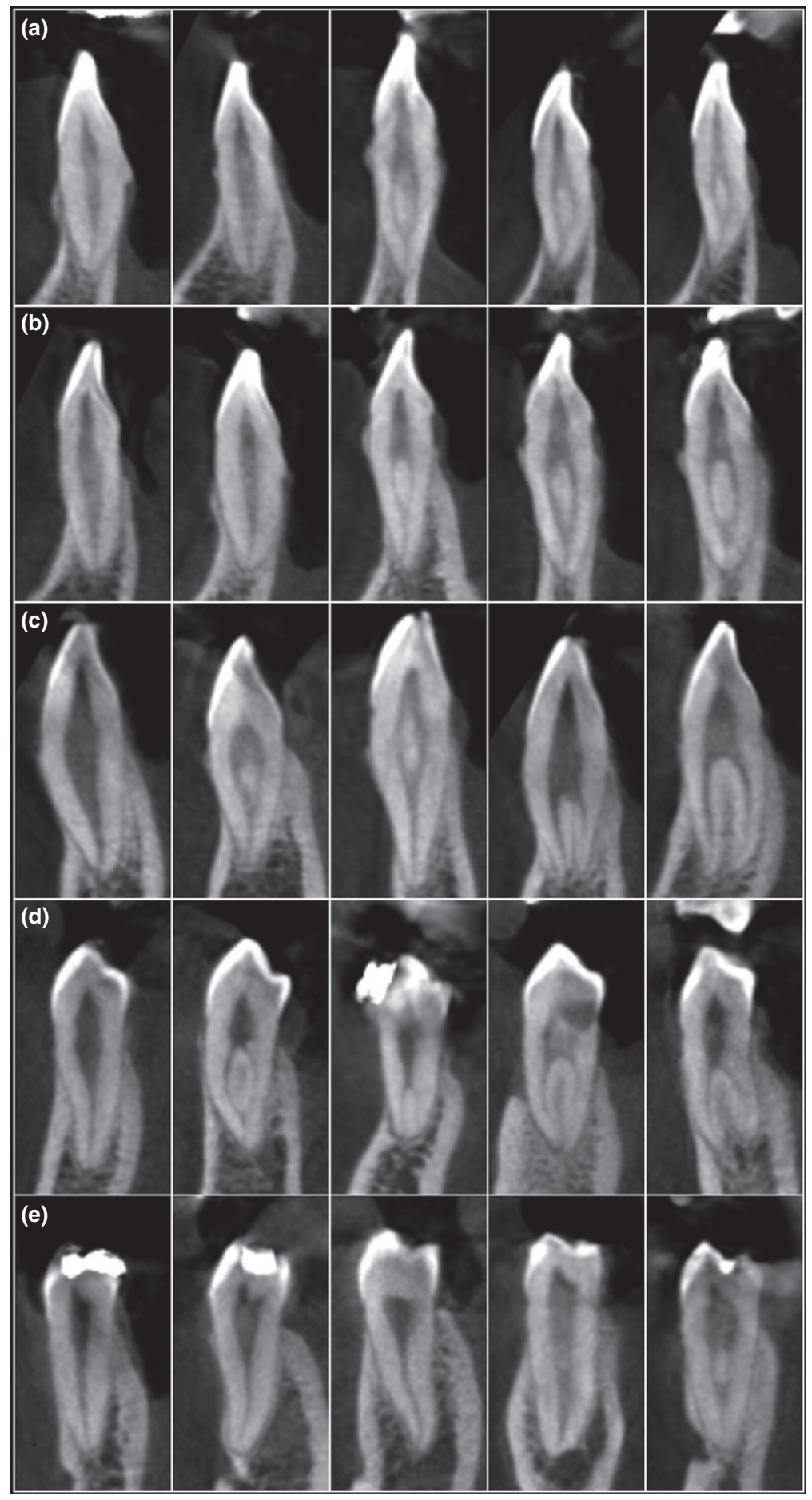

Figure 3 Examples of several root canal system configurations on mandibular teeth. (a) Central incisors, (b) lateral incisors, (c) canine, (d) first premolar, (e) second premolar. 

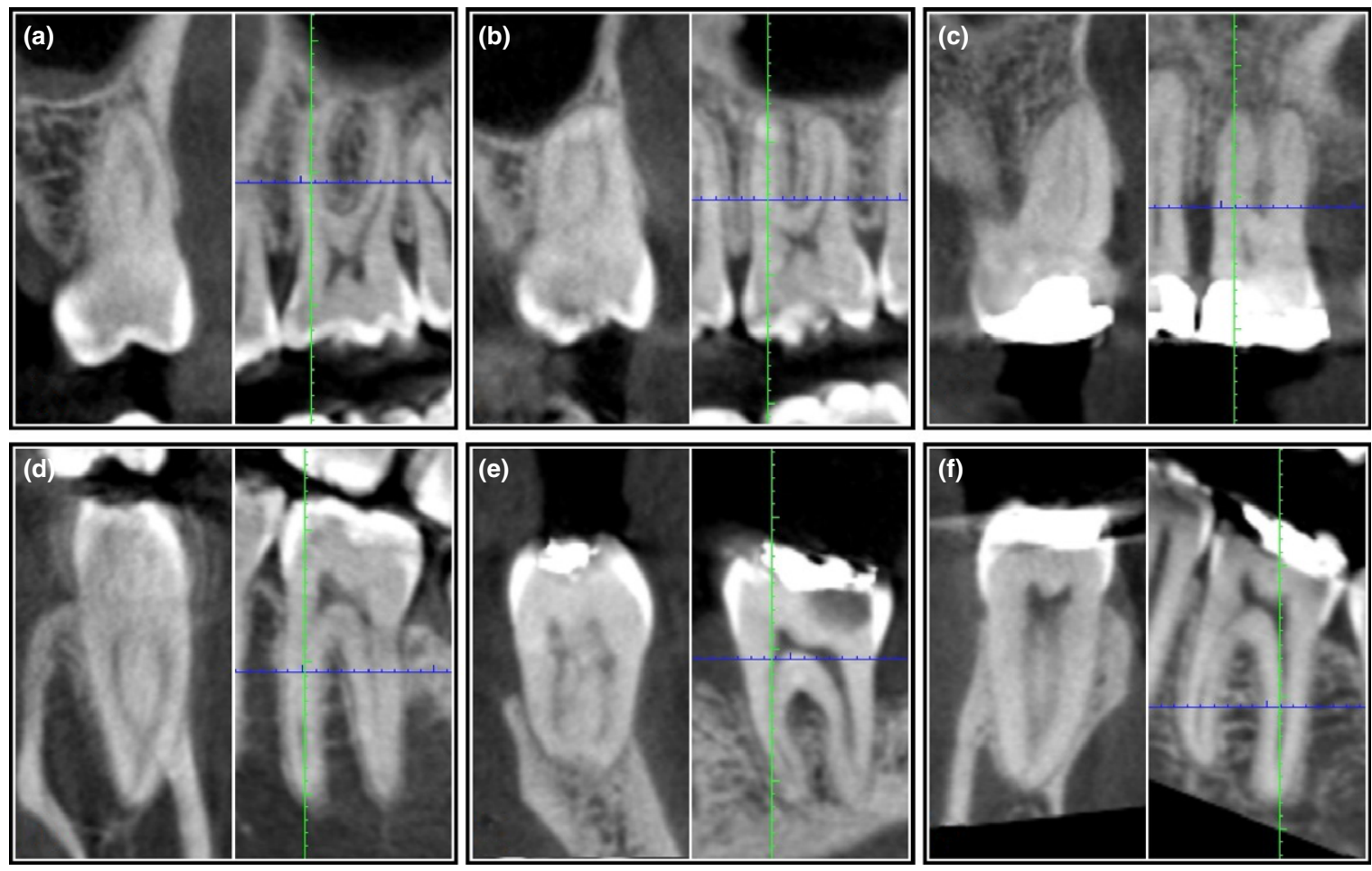

Figure 4 Root canal system morphologies on maxillary (a,b,c) and mandibular (d,e,f) molars. Due to the multiplanar angulations of some roots, the anatomy analysis had to be performed by portions using the three planes. The sagittal views correspond to the green line.

95\%]). Separation in the apical third was the most common feature in this tooth (Table 5).

Root canal systems with merging root canals were common in the maxillary second premolar $(38.7 \%$ [34.77-42.63\% CI 95\%]) and in the mesiobuccal roots of the maxillary first and second molars $(52.6 \%$ [48.2-57.0\% CI 95\%] and 32.8\% [28.94-36.66\% CI $95 \%]$, respectively). The middle level was the most common position of merging in maxillary teeth (Table 5).

An analysis of all maxillary teeth combined $(n=5078)$ revealed that 5.5\% [4.87-6.13\% CI 95\%] of maxillary teeth had root canals that split at some point, whereas $17.4 \%$ [16.36-18.44\% CI 95\%] had some type of merging. This difference was significant $(P<0.05)$.

Table 6 summarizes the main root canal splitting and merging levels of mandibular teeth. Almost 20\% of mandibular incisors and mandibular first premolars had split root canals (Table 6). Split root canals were uncommon in all of the other roots. The root canal level varied and did not exhibit a global trend in mandibular teeth.

Root canal configurations with merging root canals were common in the mesial roots of both the mandibular first and second molars (60.0\% [55.41$64.59 \%$ CI $95 \%]$ and $73.3 \%$ [69.62-76.98\% CI $95 \%]$, respectively). It was also common to find this type of anatomic feature in both the mandibular incisor and the distal roots of mandibular first molars; the prevalence was above 23\% (Table 6).

For all of the mandibular teeth combined $(n=6.429)$, the proportion of teeth with root canal splitting was $15.7 \% \quad[14.81-16.59 \% \quad$ CI $\quad 95 \%]$, whereas the proportion of mandibular teeth with merging root canals was $25.5 \%$ [24.43-26.57\% CI $95 \%]$. This difference was considered significant $(P<0.05)$. Moreover, the proportion of root canal configurations with splitting was significantly higher $(P<0.05)$ in the mandibular molars compared to the maxillary molars. Similar conditions were observed with root canal merging. 


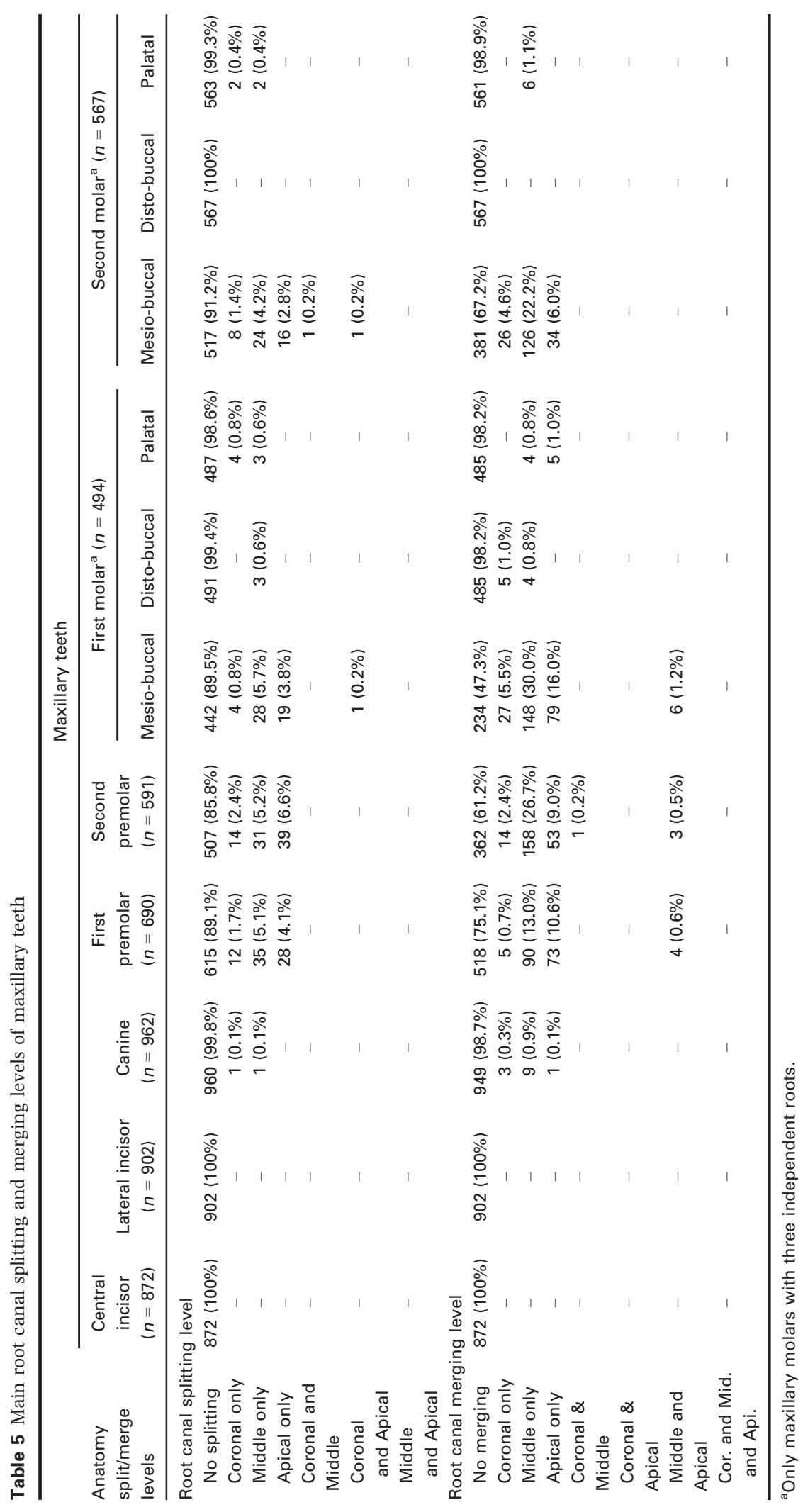




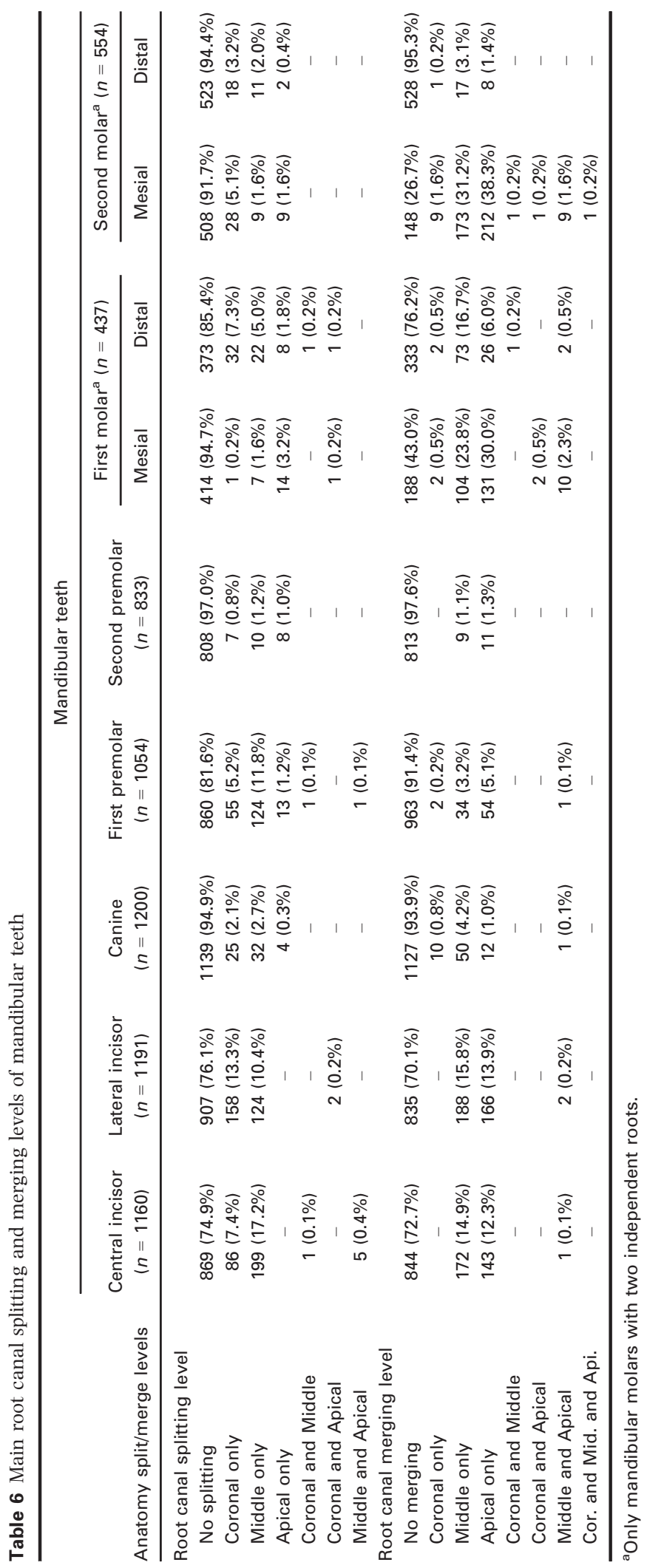




\section{Discussion}

The complexity of root canal treatment is directly associated with the number of root canals and the position where a possible split or merge may occur. Knowing that a particular main root canal splits into two at a certain point is important. A root canal split in the coronal portion of the root may be easy to locate and examine, but an abrupt separation in the middle third of the root canal may be difficult to manage, stress endodontic instruments (Pruett et al. 1997, Günday et al. 2005) and would be more difficult to fill (Hermann \& Hülsmann 2009); a split in the apical third may go unnoticed. Moreover, the success of treatment may be dependent on the awareness of these factors and any additional knowledge gained on this subject will have clinical importance.

The few studies that approached this subject (Zhang et al. 2014, Martins et al. 2016) only analysed molars with fused roots and only root canal merging. The purpose of those investigations (Zhang et al. 2014, Martins et al. 2016) was to understand if there was any relationship between root canals (of fused roots) that usually are present on separated roots. Both studies reported a high incidence of root canal merging in the apical area, but merging did occur at all levels of the root canal. One of the main objectives of present research was to understand the level of the main root canal at which merging and splitting were the most common in each group of teeth excluding molar with fused roots.

Another objective of this investigation was to understand root canal configuration using the Vertucci's classification system for all groups of teeth (the sample was collected from a population with a vast majority of Caucasians individuals). Studies that have collected data on all groups of teeth are uncommon (Vertucci 1984, Çaliskan et al. 1995, Sert \& Bayirli 2004) and have mainly used ex vivo clearing techniques. Other ex vivo techniques, such as microCT, have been used to study the root canal system anatomy (Fan et al. 2004, Zhang et al. 2014, Ordinola-Zapata et al. 2015b). The in vivo studies performed using CBCT usually analyse a particular group of teeth, but not all of them together (Silva et al. 2013, Yang et al. 2013). In this study, an attempt was made to include all groups of teeth (excluding third molars) to develop a true in vivo large sample prevalence study whilst excluding the difficulty of comparing studies with different observers or methodologies.
Root and root canal anatomy variations may be related to geographic region and ethnicity. Although the percentages may vary, the most common root canal configurations for first and second premolars are Vertucci Type IV (Abella et al. 2015 [Spain], Felsypremila et al. 2015 [India]) and Type I (Abella et al. 2015 [Spain], Bulut et al. 2015 [Turkey], Felsypremila et al. 2015 [India]), respectively. The present findings corroborate with these results. The maxillary second premolar had a higher ratio of root canal merging and splitting, and its anatomy should not be seen as less complex than that of the maxillary first premolar. The mesiobuccal roots of both maxillary molars had greater variability in the types of root canal configurations. The mesiobuccal root of the maxillary first molar with three independent roots was more prone to exhibit two root canals $(71.05 \%)$. These data are in line with the results obtained in Turkey (Altunsoy et al. 2015), India (Felsypremila et al. 2015), the United States (Guo et al. 2014) and in Asiatic countries such as Korea (Kim et al. 2012) and China (Zhang et al. 2011b, Tian et al. 2016). Other studies from Brazil (Silva et al. 2014), Italy (Plotino et al. 2013) and India (Neelakantan et al. 2010 ) reported prevalences of a single root canal (Vertucci Type I) in $52.87 \%, 59.7 \%$ and $51.8 \%$ of cases, respectively. In the mesiobuccal root of the maxillary second molar, the single root canal appears to be the most common configuration (Zhang et al. 2011b [China], Kim et al. 2012 [Korea], Neelakantan et al. 2010 [India], Plotino et al. 2013 [Italy], Altunsoy et al. 2015 [Turkey], Tian et al. 2016 [China] and the present study [Portugal]) although one study report a higher prevalence of MB2 root canals in India (Felsypremila et al. 2015). In the present study, the mandibular central and lateral incisors had a prevalence of Vertucci Type I configurations of $72.3 \%$ and $69.9 \%$, respectively, which represents a higher ratio when compared with the results from Turkey (Arslan et al. 2015), with $51.9 \%$ and $52.8 \%$, respectively, or Brazil (Estrela et al. 2015a) with 65\% and 58\%, respectively. The most common root canal configurations in the mesial root of mandibular first molars appear to be Vertucci Types II and IV (Zhang et al. 2011a, Kim et al. 2013 and the present study) or Type III (Torres et al. 2015). The mandibular first molar had two roots in $97.1 \%$ of the cases. This result was in line with other studies from India (Felsypremila et al. 2015), Italy (Plotino et al. 2013), Chile (Torres et al. 2015) and Belgium (Torres et al. 2015). However, this was a higher prevalence when compared to individuals from Korea (Kim et al. 2013) and China (Zhang et al. 2011a), 
where three roots were present in $25.85 \%$ and $29 \%$ of cases, respectively. The mandibular second molar had a single root in $14.2 \%$ of the cases, which is lower ratio when compared to data from Korea (41\%; Kim et al. 2016) or China (22\%; Zhang et al. 2011a).

One of the limitations of this research was the use of a technology (CBCT) that is not able to provide highly detailed images compared to techniques such as micro-CT or clearing (Sert \& Bayirli 2004, Ordinola-Zapata et al. 2015a,b). These techniques have been used to study small structures, such as apical foramina or isthmus (Zhang et al. 2014, OrdinolaZapata et al. 2015b). CBCT, which has a lower resolution compared to micro-CT, is unable to capture these very small anatomic details. However, the goal of the present research was to study the main root canal configuration and the merging and splitting positions in the root canal. In addition, in vivo studies are more useful when investigating the real prevalence of the anatomy, and CBCT is a technology that could be used in a routine clinic. The data collected by these imaging devices could have an impact when treating real patients. Another limitation of this study was the fact that most of the patients did not have all of their teeth. This would be interesting data to obtain, but it is very difficult to obtain information regarding all teeth unless examinations are performed for research purposes in patients with full dentition, which was not the case. Otherwise, patients usually undergo this examination when problems appear, and most often they already have missing teeth. However, a large sample of every single group of teeth was used to attempt to compensate for this limitation. The examinations were collected at a $0.20-\mathrm{mm}$ voxel size, which is a size that has previously been used successfully to study internal root canal anatomy (Helvacioglu-Yigit \& Sinanoglu 2013, Silva et al. 2013). All of the data were collected from a previously existing imaging database, which allowed the collection of a large sample size for all of the teeth without unnecessarily exposing the patients to radiation.

\section{Conclusions}

Clinicians should be aware of the position where root canals split and merge. In maxillary teeth, root canal merging occurred more often in the middle section, whereas in mandibular teeth, merging occurred more often in the middle and apical sections. Merging was more common than splits in both maxillary and mandibular teeth, and both merges and splits were more common in mandibular teeth. The roots that had a higher variability of root canal configuration types were the mesiobuccal roots of both maxillary molars, the mesial roots of the mandibular first molar and the maxillary second premolar. Extra roots, such as radix paramolaris and radix entomolaris, were rare occurrences in this Caucasian population.

\section{Conflict of interest}

The authors have stated explicitly that there are no conflicts of interest in connection with this article.

\section{References}

Abella F, Teixidó L, Patel S, Sosa F, Duran-Sindreu F, Roig M (2015) Cone-beam computed tomography analysis of the root canal morphology of maxillary first and second premolars in Spanish population. Journal of Endodontics 41, 1241-7.

Alavi AM, Opasanon A, Ng YL, Gulabivala K (2002) Root and canal morphology of Thai maxillary molars. International Endodontic Journal 35, 478-85.

Altunsoy M, Ok E, Nur B, Aglarci O, Gungor E, Colak M (2015) Root canal morphology analysis of maxillary permanent first and second molars in a southeastern Turkish population using cone-beam computed tomography. Journal of Dental Sciences 10, 401-7.

Arslan H, Ertas H, Ertas E, Kalabalik F, Saygili G, Capar I (2015) Evaluating root canal configuration of mandibular incisors with cone-beam computed tomography in a Turkish population. Journal of Dental Sciences 10, 359-64.

Bulut D, Kose E, Ozcan G, Sekerci A, Canger E, Sisman Y (2015) Evaluation of root morphology and root canal configuration of premolars in the Turkish individuals using cone beam computed tomography. European Journal of Dentistry 9, 551-7.

Çaliskan M, Pehlivan Y, Sepetçioglu TM, Tuncer S (1995) Root canal morphology of human permanent teeth in a Turkish population. Journal of Endodontics 21, 200-4.

Estrela C, Bueno M, Couto G et al. (2015a) Study of root canal anatomy in human permanente teeth in a subpopulation of Brazil's center region using cone-beam computed tomography - Part 1. Brazilian Dental Journal 26, 530-6.

Estrela C, Rabelo L, de Souza J et al. (2015b) Frequency of root canal isthmi in human permanent teeth determined by cone-beam computed tomography. Journal of Endodontics 41, 1535-9.

Fan B, Cheung GSP, Fan M, Gutmann JL, Bian Z (2004) Cshaped canal system in mandibular second molars: part I - anatomical features. Journal of Endodontics 30, 899-903.

Felsypremila G, Vinothkumar T, Kandaswamy D (2015) Anatomic symmetry of root and root canal morphology of posterior teeth in Indian subpopulation using cone beam 
computed tomography: a retrospective study. European Journal of Dentistry 9, 500-7.

Gulabivala K, Opasanon A, Ng Y-L, Alavi A (2002) Root and canal morphology of Thai mandibular molars. International Endodontic Journal 35, 56-62.

Günday M, Sazak H, Garip Y (2005) A comparative study of three different root canal curvature measured techniques and measuring the canal access angle in curved canals. Journal of Endodontics 31, 796-8.

Guo J, Vahidnia A, Sedghizadeb P, Enciso R (2014) Evaluation of root and canal morphology of maxillary permanente first molar in North America population by conebeam computed tomography. Journal of Endodontics 40, 635-9.

Helvacioglu-Yigit D, Sinanoglu A (2013) Use of cone-beam computed tomography to evaluate C-shaped root canal systems in mandibular second molars in a Turkish subpopulation: a retrospective study. International Endodontic Journal 46, 1032-8.

Hermann H, Hülsmann M (2009) Problems of root canal obturation. In: Hülsmann M, Schäfer E, eds. Problems in endodontics - etiology, diagnosis and treatment, 1st edn. London, UK: Quintessence Publishing, pp. 293-333.

Kim Y, Lee S, Woo J (2012) Morphology of maxillary first and second molars analysed by cone-beam computed tomography in a Korean population: variations in the number of roots and canals and the incidence of fusion. Journal of Endodontics 38, 1063-8.

Kim S, Kim B, Woo J, Kim Y (2013) Morphology of mandibular first molars analysed by cone-beam computed tomography in Korean population: variation in the number of roots and canals. Journal of Endodontics 39, 1516-21.

Kim S, Kim B, Kim Y (2016) Mandibular second molar root canal morphology and variants in a Korean subpopulation. International Endodontic Journal 49, 136-44.

Martins JNR, Mata A, Marques D, Caramês J (2016) Prevalence of root fusions and main root canal merging in human upper and lower molars - a CBCT in vivo study. Journal of Endodontics 42, 900-8.

Neelakantan P, Subbarao C, Ahuja R, Subbarao C, Gutmann J (2010) Cone-beam computed tomography study of root and canal morphology of maxillary first and second molars in an Indian population. Journal of Endodontics 36, 1622-7.

Ordinola-Zapata R, Bramante C, Versiani M et al. (2015a) Comparative accuracy of the clearing technique, $\mathrm{CBCT}$ and micro-CT methods in studying mesial root canal configuration of mandibular first molars. International Endodontic Journal doi: 10.1111/iej.12593. [Epub ahead of print].

Ordinola-Zapata R, Monteiro Bramante C, Gagliardi Minotti P et al. (2015b) Micro-CT Evaluation of C Shaped mandibular first premolars in a Brazilian subpopulation. International Endodontic Journal 48, 807-13.

Plotino G, Tocci L, Grande N et al. (2013) Symmetry of root and root canal morphology of maxillary and mandibular molars in a white population: a cone-beam computed tomography study in vivo. Journal of Endodontics 39, 1545-8.

Pruett J, Clement D, Carnes D (1997) Cyclic fatigue testing of nickel-titanium endodontic instruments. Journal of Endodontics 23, 77-85.

Sert S, Bayirli G (2004) Evaluation of the root canal configuration of the mandibular and maxillary permanent teeth by gender in the Turkish population. Journal of Endodontics 30, 391-8.

Silva E, Nejaim Y, Silva A, Haiter-Neto F, Cohenca N (2013) Evaluation of root canal configuration of mandibular molars in a Brazilian population by using cone-beam computed tomography: an in vivo study. Journal of Endodontics 39, 849-52.

Silva E, Nejaim Y, Silva A, Haiter-Neto F, Zaia A, Cohenca N (2014) Evaluation of root canal configuration of maxillary molars in a Brazilian population using cone-beam computed tomographic imaging: an in vivo study. Journal of Endodontics 40, 173-6.

Tian X, Yang X, Qian L, Wei B, Gong Y (2016) Analysis of the root and canal morphologies in maxillary first and second molars in a Chinese population using cone-beam computed tomography. Journal of Endodontics 42, 696-701.

Torres A, Jacobs R, Lambrechts P et al. (2015) Characterization of mandibular molar root and canal morphology using cone beam computed tomography and its variability in Belgian and Chilean population samples. Imaging Science in Dentistry 45, 95-101.

Vertucci FJ (1984) Root canal anatomy of the human permanent teeth. Oral Surgery 58, 589-99.

Yang H, Tian C, Li G, Yang L, Han X, Wang Y (2013) A cone-beam computed tomography study of the root canal morphology of mandibular first premolars and the location of root canal orifices and apical foramina in a Chinese subpopulation. Journal of Endodontics 39, 435-8.

Zhang R, Wang H, Tian Y-Y, Yu X, Hu T, Dummer P (2011a) Use of cone-beam computed tomography to evaluate root and canal morphology of mandibular molars in Chinese individuals. International Endodontic Journal 44, 990-9.

Zhang R, Yang $\mathrm{H}, \mathrm{Yu} \mathrm{X}$, Wang $\mathrm{H}, \mathrm{Hu} \mathrm{T}$, Dummer $\mathrm{P}$ (2011b) Use of CBCT to identify the morphology of maxillary permanent molar teeth in a Chinese subpopulation. International Endodontic Journal 44, 162-9.

Zhang Q, Chen H, Fan B, Fan W, Gutmann J (2014) Root and root canal morphologies in maxillary second molar with fused root from a native Chinese population. Journal of Endodontics 40, 871-5. 